\title{
Panax Notoginseng Saponins Reverse Steroid Resistance in Lupus Nephritis: Involvement of the Suppression of Exosomal P-gp Levels From Lymphocytes to Glomerular Endothelial Cells
}

\section{Feng Pan}

The Second Clinical Medical College of Zhejiang Chinese Medical University

Ying Lu ( $\sim 201811010612024 @ z c m u . e d u . c n$ )

Zhejiang Academy of Traditional Chinese Medicine

\section{Research Article}

Keywords: Panax notoginseng saponins, lupus nephritis, microangiopathy, steroid resistance, exosomes, P-gp

Posted Date: December 2nd, 2021

DOI: https://doi.org/10.21203/rs.3.rs-1100451/v1

License: (c) (i) This work is licensed under a Creative Commons Attribution 4.0 International License. Read Full License 


\section{Abstract}

Backgrounds: Microangiopathy is the most basic pathological manifestation of lupus nephritis (LN), and glomerular endothelial cells (GECs) injury is an important pathological mechanism. LN patients with microangiopathy are prone to steroid resistance (SR). Our previous studies confirmed that Panax notoginseng saponins (PNS) could reverse SR by downregulating the expression of P-gp in SR lymphocytes of LN mice (SLCsL/S). However, the mechanism of how circulating lymphocytes transmit SR information to GECs and thus affect the efficacy of kidney treatment is not clear. Recent studies have found that exosomes (exos) are an important carrier for intercellular bioactive substance communication. The aim of the study is to investigate whether exosomes derived from SLCsL/S mediate SR in GECs and PNS intervention.

Methods: Exosomes isolated from SLCsL/S were characterized, and in vitro cell coculture was further conducted to investigate the effect of SLCsL/S-derived exosomes in the SR of GECs and PNS intervention. Sequencing was used to define the exosomal miRNA expression profiling of SR GECs. Moreover, the in vivo experiments were performed through the injection of exosomes extracted from SLCsL/S into the tail vein of mice.

Results: In this study, we showed that exosomes derived from SLCsL/S could transmit SR information to GECs and lead to the aggravation of inflammatory injury through conferring P-gp, which were negated by a P-gp inhibitor. Further, we identified higher levels of exosomal miR-125b-5p from SR GECs were associated with SR in LN and could serve as biomarker for the risk of developing SR. PNS could reverse the SR of GECs and alleviate inflammatory injury by suppressing exosomal P-gp levels from lymphocytes to GECs in vitro and in vivo.

Conclusions: Our findings suggest that exosomal transfer of SLCsL/S derived P-gp confer SR to GECs, and PNS can target exosome communication to reverse SR in LN, which provides new ideas and a scientific basis for improving the clinical efficacy of traditional Chinese medicine in the treatment of refractory $L N$.

\section{Background}

Lupus nephritis $(\mathrm{LN})$ is the most common and serious complication of systemic lupus erythematosus (SLE) and is associated with considerable morbidity and even mortality[1]. Microangiopathy is the basic pathological manifestation of $\mathrm{LN}$, which often predicts more serious renal function damage and is considered to be an independent risk factor for poor renal prognosis[2]. The key pathogenesis of LN microangiopathy is the activation and injury of glomerular endothelial cells (GECs) [3, 4]. LN patients with microangiopathy have a poor response to treatment and need higher doses of steroids, showing insensitivity to steroids or even steroid resistance (SR)[5].

P-glycoprotein (P-gp), a transmembrane protein with a molecular mass of $170 \mathrm{kDa}$, is produced by a multidrug resistance gene (MDR1)[6]. P-gp is ATP-dependent and can efflux drug substrates to maintain 
appropriate intracellular levels. The overexpression of P-gp is one key mechanism contributing to SR[7, 8]. Our previous unpublished studies confirmed that silent information regulation factor related enzyme 1 (SIRT1) in SR splenic lymphocytes of LN mice (SLCsL/S) initiates the transcription of MDR1 by upregulating the nuclear transcription factor forkhead box-containing protein of the 0 subfamily 1 (Fox01), resulting in high expression of P-gp. However, the mechanism by which circulating lymphocytes transmitting drug resistance information to GECs producing SR is unknown.

Recent studies have shown that cells can communicate with adjacent or distant cells through the secretion of exosomes (exos), which are spherical nanovesicles encapsulated by bilayer membranes with a diameter of 30-150 nm and carry a variety of functional substances in the source cells, including proteins, lipids and miRNAs[9-11]. Exosomes expelled from drug-resistant cells expressing high levels of P-gp and can deliver the same pattern of P-gp to sensitive cells, which may be a mechanism of exosomemediated drug resistance[12-14]. miRNAs, a conserved non-coding RNAs, have been found to be closely related to drug resistance. Secreted miRNAs, especially those in exosomes, have natural advantages due to their stable structure and minimal non-specific interference and may serve as better biomarkers to detect SR in LN[15]. Therefore, SLCsL/S may transmit SR signals (such as P-gp) to GECs to produce drug resistance through exosomes. In addition, exosomal miRNAs secreted by SR GECs may be a biomarker for the risk of developing SR in LN.

Panax notoginseng saponins (PNS) is a chemical component extracted from Panax notoginseng herbal medicine that contains a variety of different monomers, such as notoginsenoside R1, ginsenoside Rg1, and ginsenoside Re[16]. It can anti-inflammatory, antioxidative stress and protects vascular endothelial cells[17-19]. Most notably, PNS has an obvious ability to reverse multiple drug resistance including P-gpmediated multidrug resistance in many studies regarding animals and cells[20-23]. Our previous studies confirmed that PNS could significantly downregulate the levels of P-gp in patients with LN, increase the accumulation of steroids in cells, and synergistically enhance their immunosuppressive effect, which has more advantages than the P-gp inhibitor verapamil[24]. Previous unpublished studies further confirmed that PNS could reverse SR in SLCsL/S through the SIRT1/P-gp pathway. However, it remains unclear whether PNS can reverse the SR of GECs and alleviate microangiopathy by suppressing exosomal P-gp levels from lymphocytes to GECs.

In this study, we confirmed that P-gp-containing exosomes from SLCsL/S could cause GECs to acquire SR and further aggravate inflammatory injury through conferring P-gp. Exosomal miR-125b-5p from SR GECs could be used as predictors for detecting SR in LN. Further investigation demonstrated that PNS could reverse the SR of GECs and alleviate inflammatory injury by suppressing exosomal P-gp levels from lymphocytes to GECs. This study provides a theoretical basis for PNS in the treatment of refractory LN.

\section{Methods}

\section{Establishment and grouping of the animal model}


Female NZB/WF1 and ICR mice (specific pathogen free, SPF) aged 16 weeks were acquired from Jackson Laboratory by the iBio Logistics Co., Ltd. (Beijing, China) and the Zhejiang Academy of Traditional Chinese Medicine, respectively, and were housed in accordance with relevant guidelines. All procedures involving mice were approved by the Animal Ethics Committee of Zhejiang Academy of Traditional Chinese Medicine (approval number: ZATCM-2019045) and were carried out in accordance with the ARRIVE guidelines.

The SR lupus mouse (SRLM) model was established by the intraperitoneal injection of low-dose Meyhylprednisolone(MP, Pfizer, New York, USA) for eight weeks $\left(0.8 \mathrm{mg} \cdot \mathrm{kg}^{-1} \cdot \mathrm{day}^{-1}\right)$ according to our previous study[25]. Compared with untreated mice, the criterion for the successful construction of the SRLM model was that the expression of P-gp increased and the accumulation of Rh-123 decreased in the splenic lymphocytes of LN mice (SLCsL)(Supplementary Fig. 1). ICR mice were used as a control group (normal saline, 8 weeks). NZB/WF1 mice were randomly divided into the LN group (normal saline, 8 weeks), SR group (0.8 $\mathrm{mg} \cdot \mathrm{kg}^{-1} \cdot$ day $^{-1} \mathrm{MP}, 8$ weeks), SR with PNS (Guangxi Wuzhou Pharmaceutical Co., Ltd, Guangxi, China) (Supplementary Fig. 2) group (0.8 mg $\mathrm{kg}^{-1} \cdot \mathrm{day}^{-1} \mathrm{MP}, 100 \mathrm{mg} \cdot \mathrm{kg}^{-1} \cdot \mathrm{day}^{-1} \mathrm{PNS}, 8$ weeks) and SR with P-gp inhibitor Tariquidar (Tariq, Selleckchem, TX, USA) group (0.8 $\mathrm{mg} \cdot \mathrm{kg}^{-1} \cdot \mathrm{day}^{-1} \mathrm{MP}, 8 \mathrm{mg} \cdot \mathrm{kg}^{-}$ ${ }^{1} \cdot$ day $^{-1}$ Tariq, 8 weeks).

\section{Preparation of splenic lymphocytes}

After the early intervention, five groups of mice were euthanized through $\mathrm{CO}_{2}$ asphyxiation using slow displacement of chamber air with compressed $\mathrm{CO}_{2}(25 \% / \mathrm{min})$, and their splenic lymphocytes were extracted, namely, the control group (SLCsC), LN group (SLCsL), SR group (SLCsL/S), SR with PNS group (SLCsL/S+PNS), and SR with Tariq group (SLCsL/S+Tariq). Lymphocytes were isolated and cultured as previously described[26].

\section{Preparation of GECs}

The GECs of ICR and NZB/WF1 mice were separated as previously described[27] and cultured in endothelial cell medium supplemented with $10 \% \mathrm{FBS}$ at $37^{\circ} \mathrm{C}$ with $5 \% \mathrm{CO}_{2}$.

\section{Cell co-culture assay}

SLCsC and SLCsL, SLCsL/S, SLCsL/S+Tariq $\left(5 \times 10^{5} /\right.$ well $)$ were seeded into the upper chamber of a coculture system and GECs $\left(3 \times 10^{5} /\right.$ well $)$ from ICR and NZB/WF1 mice were placed in the lower chamber and incubated for $48 \mathrm{~h}$. Co-cultured cells were separated using Transwell permeable supports $(0.4 \mu \mathrm{m})$ in poly carbonate membrane(Costar, Cambridge, MA, USA).

\section{Isolation of exosomes}

Exosomes were isolated by ultracentrifugation as described previously[28]. In brief, the culture supernatants of cells were collected and sequentially centrifuged at $300 \mathrm{~g}$ for $10 \mathrm{~min}, 2000 \mathrm{~g}$ for $15 \mathrm{~min}$, 
and $10,000 \mathrm{~g}$ for $30 \mathrm{~min}$ to remove cell debris and large vesicles, and then, the cleared sample was further ultracentrifuged for $2 \mathrm{~h}$ at $100,000 \mathrm{~g}$ to pellet the exosomes. The extracted exosomes were allocated and stored at $-80{ }^{\circ} \mathrm{C}$ or used for downstream experiments.

\section{Transmission electron microscopy (TEM)}

The morphology of exosomes was observed by TEM[29]. After absorbing onto Formvarcarbon-coated copper mesh grids, exosomes were negatively stained with aqueous phosphotungstic acid for 1-2 min. After air dry, samples were visualized with a Hitachi 7100 transmission electron microscope.

\section{Nanoparticle-tracking analysis (NTA)}

We measured the exosome particle size and concentration using nanoparticle tracking analysis (NTA) at VivaCellBiosceinces with ZetaView PMX 110 (Particle Metrix, Meerbusch, Germany)[30]. Isolated exosome samples were appropriately diluted using 1X PBS buffer (Biological Industries, Israel) to measure the particle size and concentration. NTA measurement was recorded and analyzed at 11 positions. The ZetaView system was calibrated using $110 \mathrm{~nm}$ polystyrene particles. Temperature was maintained around $23^{\circ} \mathrm{C}$ and $30^{\circ} \mathrm{C}$.

\section{Western blot analysis}

Exosomes were collected, and total protein was extracted and quantified using a BCA protein assay kit (Beyotime Biotechnology, Shanghai, China). Next, the immunoreactive proteins were detected using the Simon ${ }^{\mathrm{TM}}$ machine (ProteinSimple, San Jose, USA). The primary and secondary antibodies used were the following: rabbit anti-CD63 antibody(Abcam, USA), anti-TSG101 antibody(Abcam), anti-Calnexin antibody(Abcam), anti-P-gp antibody(Abcam) and anti- $\beta$-actin antibody(Cell Signaling Technology, USA), anti-rabbit IgG(ProteinSimple). Quantization of detected proteins and image preparation were performed with Compass Software (ProteinSimple).

\section{Exosome internalization}

Exosomes were labeled with the green lipophilic fluorescent dye PKH67 (Sigma-Aldrich, St. Louis, MO) according to the manufacturer's instructions. After coculture with PKH67-stained exosomes for $24 \mathrm{~h}$, GECs were stained with DAPI (Abcam) for the nucleus and phalloidin (Abcam) for the intracellular cytoskeleton F-actin according to the instruction manual and observed under a confocal laser microscope.

\section{Flow cytometry}

GECs were trypsinized and washed twice in $1 \times$ PBS. After resuspension in $100 \mu \mathrm{l}$ of $1 \times$ PBS, FITCconjugated antibodies against P-gp (Abcam) and Rh-123 (Sigma) or their respective isotype controls were stained for $30 \mathrm{~min}$ at $4{ }^{\circ} \mathrm{C}$. Following washing twice in $1 \times$ PBS, labeled cells were measured by flow cytometry (BD Biosciences) and analyzed with FlowJo software (Tree Star). 


\section{Dexamethasone accumulation}

After exosomes intervention, GECs were washed twice in $1 \times$ PBS and then incubated with $3.0 \times 10^{-8} \mathrm{M}^{3} \mathrm{H}$ labeled dexamethasone for 20 minutes at $37^{\circ} \mathrm{C}$. The intracellular dexamethasone concentration was counted with a scintillation counter, according to previously study[31].

\section{GECs exosomal miRNA sequencing}

The total RNA was extracted using the Total RNA Purification Kit (LC Sciences, Houston, USA), according to the manufacturer's protocol. The total RNA quantity and purity were analysis of Bioanalyzer 2100 and RNA 6000 Nano LabChip Kit (Agilent, CA, USA) with RIN number >7.0. Then, the first strand cDNA was synthesized and PCR amplification was performed according to protocol of TruSeq Small RNA Sample Prep Kits(Illumina, San Diego, USA), and Samll RNAs were used for library preparation. Finally, we performed the single-end sequencing (1x50 bp) on an Illumina Hiseq2500 at the LC-BIO (Hangzhou, China) following the vendor's recommended protocol[32].

\section{Animal experiments}

To determine the role of SLCsL/S exosomes and PNS in the SR and inflammatory injury of GECs in vivo, 16-weeks-old female ICR and NZB/WF1 mice were injected via the tail vein with exosomes from lymphocytes. ICR mice were used as a control group: SLCsC-exo group $(n=6,5 L C s C$-derived exosomes treatment). NZB/WF1 mice were randomly divided into four groups: SLCsL-exo group ( $n=6, \mathrm{SLCsL}$-derived exosomes treatment), SLCsL/S-exo group ( $n=6, S L C s L / S$-derived exosomes treatment), SLCsL/S+PNSexo group ( $n=6, S L C s L / S+P N S$-derived exosomes treatment), and SLCsL/S+Tariq-exo group ( $n=6$, SLCsL/S+Tariq-derived exosomes treatment). All the groups were injected five times every week for three consecutive weeks and then the serum, urine, and renal cortex samples were harvested for further analysis.

\section{Enzyme-linked immunosorbent assay (ELISA)}

GECs supernatant and serum samples were centrifuged at $3500 \times \mathrm{g}$ for $10 \mathrm{~min}$ at $4{ }^{\circ} \mathrm{C}$. The concentrations of IL-1 $\beta$, IL-6, MCP-1, VCAM-1, ANA and anti-dsDNA were detected by using ELISA kits according to the manufacturer's instructions.

\section{Real-time PCR analysis}

The total RNA of exosomes, GECs and tissues was isolated by using E.Z.N.A total RNA kit (Omega Biotech Inc., Norcross, GA, USA) according to the manufacturer's protocol and converted to cDNA with the PrimeScriptTM RT reagent kit (Takara, Dalian, China). Quantitative real-time PCR was performed using SYBR-green mix (TaKaRa) and run on a 7500 Real-Time PCR System (Applied Biosystems). The relative expression levels of mRNA and miRNA were normalized against $\beta$-actin and $\mathrm{U} 6$, analyzed using the 2- $\triangle$ Ct method. IL-1 $\beta$ forward primer: 5'-TCGCAGCAGCACATCAACAAGAG-3'; reverse primer: 5'AGGTCCACGGGAAAGACACAGG-3'. IL-6 forward primer: 5'-CTTCTTGGGACTGATGCTGGTGAC-3'; reverse 
primer: 5'-AGGTCTGTTGGGAGTGGTATCCTC-3'. MCP-1 forward primer: 5'-

CCACTCACCTGCTGCTACTCATTC-3'; reverse primer: 5'-CTTCTTTGGGACACCTGCTGCTG-3'. VCAM-1 forward primer: 5'-GAGGGTGGTGCTGTGACAATGAC-3'; reverse primer: 5'-

GGGTGGCATTTCCTGAGAGAAGC-3'. MDR1 forward primer: 5'-TGATCGCTCACCGCCTGTCC-3'; reverse primer: 5'-GTGCCGTGCTCCTTGACCTTG-3'. $\beta$-actin forward primer: 5'-TATGCTCTCCCTCACGCCATCC-3'; reverse primer: 5'-GTCACGCACGATTTCCCTCTCAG-3'. miR-125b-5p forward primer: 5'-

TGAGACCCTAACTTGTGAGTCGTATC-3'; reverse primer: 5'-CAGTGCAGGGTCCGAGGTAT-3'. miR-128-3p forward primer: 5'-GCGGTCTCTTTGTCGTATCCA-3'; reverse primer: 5'-CAGTGCAGGGTCCGAGGTA-3'. U6 forward primer: 5'-CTCGCTTCGGCAGCACA -3'; reverse primer: 5'-AACGCTTCACGAATTTGCGT-3'.

\section{Hematoxylin and eosin (HE) staining and Immunohistochemical analysis (IHC)}

To determine renal histopathology, 3- $\mu$ m-thick paraffin sections of the kidney were stained with hematoxylin and eosin (HE). Renal histology was assessed blindly by an experienced pathologist. Renal histopathological changes were quantitated as described previously[33].

After deparaffinization and rehydration, paraffin sections were blocked with Dual Endogenous Enzyme Block (DAKO, Glostrup, Denmark) for IHC. The primary antibody was rabbit anti-P-gp (or IL-1 $\beta$, IL-6, MCP-1, VCAM-1) IgG (Abcam). The secondary antibody was polymer-horseradish peroxidase-labeled goat antirabbit IgG (Abcam). Finally, sections were incubated with 3, 3'-diaminobenzine-chromogen substrate (DAKO) and counterstained with hematoxylin. The stains were scored by a renal pathologist blinded to the mice grouping.

\section{Serum and urine analyses}

The levels of $24 \mathrm{~h}$ urinary protein (24 h Upro), serum creatinine (Scr) and nitrogen concentration (BUN) were assessed using commercially available kits (Jian Cheng Bioengineering Institute, Nanjing, China) according to the manufacturer's directions.

\section{Statistical Analysis}

The analysis process was performed by SPSS 22.0 software (SPSS Inc., Chicago, IL, USA), and measurement data consistent with a normal distribution are expressed as the mean \pm standard deviation. Student's $t$-test and One-way ANOVA were used to compare differences between two or more groups. A value of $P<0.05$ indicated statistically significant differences.

\section{Results}

\section{SLCsL/S induces the SR of GECs}

The levels of P-gp and its transport substrate rhodamine 123 (Rh-123) are the main marker to judge SR[34]. To determine whether SLCsL/S can confer SR to GECs, we cocultured GECs with SLCsL/S in a Transwell insert, which prevents direct cell-to-cell contact. The results showed a significant increase in P- 
gp expression and a decrease in Rh-123 accumulation in cells cocultured with SLCsL/S compared with those cocultured with SLCsL and SLCsC (Fig. 1). In a word, these data indicated that SLCsL/S prompted the development of SR in GECs.

\section{Lymphocyte-derived exosomes can be internalized by GECs and PNS downregulates the levels of P-gp in exosomes by SLCsL/S}

Many studies have manifested that exosomes could mediate SR by transporting P-gp. To explore the function of SLCsL/S-derived exosomes on SR of GECs, exosomes from lymphocytes were isolated and identified firstly. TEM showed the presence of cup- or sphere- shaped morphology with double-membrane structures (Fig. 2A). The observation of NTA indicated that the diameter of isolated exosomes was 128 $\mathrm{nm}$, and the concentration was $1.3 \times 10^{12}$ particle/ml (Fig. 2B). WB verified the expression of the marker proteins CD63 and TSG101 in the exosomes (Fig. 2C).

In order to demonstrate lymphocyte derived exosomes could be taken in by GECs, exosomes were firstly labelled with PKH67, a fluorescent cell linker compound, which shows strong green fluorescence under the fluorescence microscope and cocultured with GECs for $24 \mathrm{~h}$. After stained nucleus and cytoskeleton Factin with DAPI and phalloidin, the uptake of lymphocyte derived exosomes by GECs was visualized with confocal microscopy. The results revealed that PKH67-labeled lymphocyte-exos were mainly localized in the cytoplasm of GECs, indicating that lymphocyte-exos could be taken up by GECs (Fig. 2D).

To determine the impact of PNS on P-gp expression in SLCsL/S-derived exosomes, the exosomal P-gp levels were measured. We found that compared with SLCsL-exos and SLCsC-exos, the expression of P-gp in SLCsL/S-exos increased, while PNS and Tariq significantly downregulated the P-gp levels in SLCsL/Sexos (Fig. 2E, F).

\section{SLCsL/S-derived exosomes induce the SR and aggravate inflammatory injury in GECs and PNS intervention in vitro}

To investigate the functional roles of SLCsL/S-exos in the SR and inflammatory injury of GECs, GECs were incubated with exosomes purified from SLCsC, SLCsL or SLCsL/S for $48 \mathrm{~h}$. First, we tested the expression of P-gp and the accumulation of Rh-123 in GECs by flow cytometry. The results show that, compared with SLCsC-exos and SLCsL-exos, the expression of P-gp was upregulated and the accumulation of Rh-123 was decreased in GECs treated with SLCsL/S-exos(Fig. 3A-D). Furthermore, we demonstrated that intracellular dexamethasone levels in GECs incubated with SLCsL/S-exos were significantly decreased(Fig. 3E). Second, ELISA and RT-PCR were performed to detect the levels of IL-1 $\beta$, IL-6, MCP-1 and VCAM-1 in GECs. As shown in Figs. 3F and 3G, incubation with exosomes released by SLCsL/S resulted in increased expression of IL-1 $\beta$, IL-6, MCP-1 and VCAM-1 in GECs at both the mRNA and protein levels compared to the SLCsC and SLCsL.

To identify differentially expressed miRNAs that change during SR, miRNA profiles assay was performed in exosomes derived from GECs treated with SLCsL-exos and SLCsL/S-exos using Illimina NextSeq 500 
technology. After comparing the miRNA profiles of the two groups, there were 3 significantly upregulated miRNAs and 3 significantly downregulated miRNAs(Fig. 3H). We chose two upregulated miRNAs for validation using RT-PCR. The results confirmed significant expression of miR-125b-5p but not miR-1283p(Fig. 3l, J).

To further determine whether PNS could reverse the SR of GECs induced by SLCsL/S-exos to alleviate the inflammatory injury of GECs, GECs were cocultured with SLCsL/S+PNS-exos for $48 \mathrm{~h}$. The data showed that the expression of P-gp was downregulated and the accumulation of Rh-123 was increased in GECs treated with SLCsL/S+PNS-exos and SLCsL/S+Tariq-exos compared with SLCsL/S-exos, and PNS could increase the levels of intracellular dexamethasone in GECs(Fig. 3A-D). Meanwhile, the protein and mRNA levels of IL-1 $\beta$, IL-6, MCP-1 and VCAM-1 were decreased in GECs treated with SLCsL/S+PNS-exos and SLCsL/S+Tariq-exos compared with SLCsL/S-exos(Fig. 3F, G).

Taken together, these results demonstrated that exosomes secreted by SLCsL/S induced GECs to acquire SR to aggravate inflammatory injury through conferring P-gp, whereas PNS could reverse the SR of GECs induced by SLCsL/S-exos and alleviate the inflammatory injury by suppressing exosomal P-gp levels from lymphocytes to GECs in vitro.

\section{SLCsL/S-derived exosomes induce the SR and aggravate inflammatory injury in GECs and PNS intervention in vivo}

We next sought to explore the effect of SLCsL/S-derived exosomes on the SR of GECs and inflammatory injury in vivo through the injection of exosomes extracted from SLCsL/S into the tail vein of mice. The results of HE staining showed that GECs proliferated with lymphocyte infiltration in the mice injected with SLCsL/S-exos compared with SLCsC-exos and SLCsL-exos, while SLCsL/S+PNS-exos and SLCsL/S+Tariq-exos could reduce the proliferation of GECs and the infiltration of lymphocytes(Fig. 4A). IHC and RT-PCR results showed that the protein and mRNA levels of P-gp, IL-1 $\beta$, IL-6, MCP-1 and VCAM-1 were significantly increased in the mice injected with SLCsL/S-exos, but these indicators were decreased when treated with SLCsL/S+PNS-exos and SLCsL/S+Tariq-exos(Fig. 4A, B). The levels of $24 \mathrm{~h}$ Upro, Scr, BUN, ANA and dsDNA were increased in the mice injected with SLCsL/S-exos, while treatment with SLCsL/S+PNS-exos and SLCsL/S+Tariq-exos could reduce their levels(Fig. 4C-G).

In summary, our data show that exosomes secreted by SLCsL/S induce kidney-acquired RS to aggravate microangiopathy, whereas PNS could reverse the SR induced by SLCsL/S-exos and alleviate kidney injury through reducing exosomal P-gp levels from lymphocytes to GECs in vivo.

\section{Discussion}

LN patients with microangiopathy are prone to SR. GECs are the main effector cells of LN microangiopathy. Our previous studies found that there was P-gp-mediated SR in SLCsL, but the mechanism of how lymphocytes transmitted drug resistance information to GECs to produce SR is not clear. Several studies have indicated the marvelous roles of exosomes in drug resistant by conferring 
functional protein including P-gp[35]. However, whether the exosomes of circulating SR lymphocytes can carry P-gp into GECs and induce SR in GECs has not been explored. In the present study, we first showed that SLCsL/S can transmit SR information to GECs. Then, we further found that exosomes derived from SLCsL/S expressed higher levels of P-gp than those derived from SLCsC and SLCsL, and these exosomes could be taken up into GECs. Exosomes extracted from SLCsL/S, but not from SLCsC and SLCsL, could confer SR to GECs by the delivery of P-gp both in vitro and in vivo. Furthermore, intracellular levels of dexamethasone were found to decrease significantly in GECs treated with SLCsL/S-Exos. Moreover, our study found that when GECs acquired SR in vivo and in vitro, their inflammatory injury will be aggravated, and they will secrete more proinflammatory cytokines.

Extensive studies indicated that dysregulation of exosomal miRNA expression has been associated with SR, which show significant potential as first biomarkers for SR and can be highly stable in body fluids[36]. To our knowledge, this is the first study investigating exosomal miRNA profiling of SR GECs in $L N$ mice. The results showed that 6 miRNAs were found to be significantly differentially expressed in the exosomal from SR GECs(3 upregulated and 3 downregulated). After validation by RT-PCR, miR-125b-5p was observed to be significantly upregulated. Therefore, exosomal miR-125b-5p can be used as predictors of steroids treatment efficacy and the patient's risk of developing SR.

To find effective substances to reverse SR, a growing number of researchers have focused their research on the exploration of the active ingredients from natural sources that have the ability to restore relevant indicators to normal levels. Many active components of traditional Chinese medicine have been shown to reverse drug resistance by restraining P-gp expression[37-40]. Our previous studies showed that PNS could inhibit the expression of P-gp without significant cytotoxicity. Taken together, that PNS might have great potential to reverse SR needed to be explored, and thus, it is logical to hypothesize that PNS could reverse the SR of GECs through inhibiting the exosome-mediated intercellular transfer of P-gp in the SLCsL/S-to-GECs direction.

In this study, we first determined the effect of PNS and Tariq on exosomal P-gp levels. The results showed significantly decreased P-gp expression in the exosomes extracted from SLCsL/S treated with PNS and Tariq. The following in vitro experiments further confirmed that compared with SLCsL/S, the expression of P-gp was downregulated and the accumulation of Rh-123 and intracellular dexamethasone were increased in GECs treated with exosomes derived from SLCsL/S+PNS and SLCsL/S+Tariq, and the levels of secreted proinflammatory factors were also reduced accordingly. This shows that PNS could reverse the SR of GECs by inhibiting the expression of P-gp in SLCS/MP-derived exosomes to alleviate inflammatory injury. This conclusion has also been further verified by in vivo experiments. In the present study, we showed that, compared with SLCsL/S-derived exosome intervention, PNS- and Tariq-treated exosomes could reduce the expression of P-gp and proinflammatory-related factors in renal tissue. At the same time, SLCsL/S exosomes treated with PNS and Tariq also alleviated renal pathological injury in mice and reduced 24-hour urinary protein, Scr, BUN, ANA and dsDNA.

\section{Conclusions}


In summary, this is the first report of a P-gp transferred from lymphocytes to GECs by the means of exosomes. Our previous data demonstrate that P-gp-enriched exosomes produced by SLCsL/S may be critical for SR of GECs in LN. Exosomal miR-125b-5p from SR GECs may serve as biomarkers in SR of LN and can be used as predictors of prognosis and steroids treatment efficacy. Meanwhile, we raise the possibility that PNS may be of therapeutic value for treating SR and alleviating microangiopathy in LN partly through reducing the content of P-gp in the exosomes transferred from SLCsL/S to GECs(Fig. 5).

\section{Abbreviations}

LN, lupus nephritis; SLE, systemic lupus erythematosus; GECs, glomerular endothelial cells; SR, steroid resistance; P-gp, P-glycoprotein; MDR1, multidrug resistance 1; SIRT1, silent information regulation factor related enzyme 1; Fox01, forkhead box-containing protein of the 0 subfamily 1 ; exos, exosomes; PNS, Panax notoginseng saponins; SLCsL/S, steroid resistance splenic lymphocytes of lupus nephritis mice; SLCsL, splenic lymphocytes of lupus nephritis mice; SLCsC, splenic lymphocytes of ICR mice; Tariq, Tariquidar; Rh-123, rhodamine-123.

\section{Declarations}

\section{Ethics approval and consent to participate}

All animal experiments were conducted in accordance with the ARRIVE guidelines and prior approval from the Animal Ethics Committee of Zhejiang Academy of Traditional Chinese Medicine (approval no. ZATCM-2019045).

\section{Consent for publication}

The authors declare that they agree to submit the article for publication.

\section{Availability of data and materials}

The datasets used and analysed during the current study are available from the corresponding author, Ying Lu, on reasonable request.

\section{Competing interests}

All authors declare that they have no conflicts of interest.

\section{Funding}

The work was supported by the National Natural Science Foundation of China (Grant numbers 81673818 , 81974549). The funding body was not involved in the design of the study; the collection, analysis, and interpretation of data; or the writing of the manuscript.

\section{Author contributions}


FP carried out the experiments and drafted the manuscript. YL designed and coordinated the study, revised the manuscript and submitted it for publication. All authors analysed and interpreted the data. The author(s) read and approved the final manuscript.

\section{Acknowledgements}

Not applicable.

\section{References}

1. Yang Y, Zhang Z, Zhuo L, Chen DP, Li WG. The Spectrum of Biopsy-Proven Glomerular Disease in China: A Systematic Review. Chin Med J (Engl). 2018; 131(6): 731-735.

2. Tan Y, Yu F, Liu G. Diverse vascular lesions in systemic lupus erythematosus and clinical implications. Curr Opin Nephrol Hypertens. 2014; 23(3): 218-23.

3. Atehortúa L, Rojas M, Vásquez GM, Castaño D. Endothelial Alterations in Systemic Lupus Erythematosus and Rheumatoid Arthritis: Potential Effect of Monocyte Interaction. Mediators Inflamm. 2017; 2017: 1-12.

4. Bajema IM, Wilhelmus S, Alpers CE, Bruijn JA, Colvin RB, Cook HT, D’Agati VD, Ferrario F, Haas $M$, Jennette JC, Joh K, Nast CC, Noël LH, Rijnink EC, Roberts ISD, Seshan SV, Sethi S, Fogo AB. Revision of the International Society of Nephrology/Renal Pathology Society classification for lupus nephritis: clarification of definitions, and modified National Institutes of Health activity and chronicity indices. Kidney Int. 2018; 93(4): 789-796.

5. Hu WX, Liu ZZ, Chen HP, Zhang HT, Li LS, Liu ZH. Clinical characteristics and prognosis of diffuse proliferative lupus nephritis with thrombotic microangiopathy. Lupus. 2010; 19(14): 1591-8.

6. Vadlapatla RK, Vadlapudi AD, Pal D, Mitra AK. Mechanisms of drug resistance in cancer chemotherapy: coordinated role and regulation of efflux transporters and metabolizing enzymes. Curr Pharm Des. 2013; 19(40): 7126-40.

7. Gao H, Wang Q, Yu X, Liu J, Bai S, Feng J, Wu B. Molecular mechanisms of glucocorticoid resistance in systemic lupus erythematosus: A review. Life Sci. 2018; 209: 383-387.

8. Perez-Guerrero EE, Gamez-Nava JI, Muñoz-Valle JF, Cardona-Muñoz EG, Bonilla-Lara D, FajardoRobledo NS, Nava-Zavala AH, Garcia-Cobian TA, Rincón-Sánchez AR, Murillo-Vazquez JD, CardonaMüller D, Vazquez-Villegas ML, Totsuka-Sutto SE, Gonzalez-Lopez L. Serum levels of P-glycoprotein and persistence of disease activity despite treatment in patients with systemic lupus erythematosus. Clin Exp Med. 2018; 18(1): 109-117.

9. Lv LL, Feng Y, Tang TT, Liu BC. New insight into the role of extracellular vesicles in kidney disease. J Cell Mol Med. 2019; 23(2): 731-739.

10. Lässer C, Jang SC, Lötvall J. Subpopulations of extracellular vesicles and their therapeutic potential. Mol Aspects Med. 2018; 60: 1-14. 
11. Huang-Doran I, Zhang CY, Vidal-Puig A. Extracellular Vesicles: Novel Mediators of Cell Communication In Metabolic Disease. Trends Endocrinol Metab. 2017; 28(1): 3-18.

12. Zhang HD, Jiang LH, Hou JC, Zhong SL, Zhu LP, Wang DD, Zhou SY, Yang SJ, Wang JY, Zhang Q, Xu $\mathrm{HZ}$, Zhao JH, Ji ZL, Tang JH. Exosome: a novel mediator in drug resistance of cancer cells. Epigenomics. 2018; 10(11): 1499-1509.

13. Namee NM, O'Driscoll L. Extracellular vesicles and anti-cancer drug resistance. Biochim Biophys Acta Rev Cancer. 2018; 1870(2): 123-136.

14. Torreggiani E, Roncuzzi L, Perut F, Zini N, Baldini N. Multimodal transfer of MDR by exosomes in human osteosarcoma. Int J Oncol. 2016; 49(1): 189-96.

15. Mori MA, Ludwig RG, Garcia-Martin R, Brandão BB, Kahn CR. Extracellular miRNAs: From Biomarkers to Mediators of Physiology and Disease. Cell Metab. 2019; 30(4): 656-673.

16. Liu Y, Zhang HG, Jia Y, Li XH. Panax notoginseng saponins attenuate atherogenesis accelerated by zymosan in rabbits. Biol Pharm Bull. 2010; 33(8): 1324-30.

17. Meng L, Lin J, Huang Q, Liang P, Huang J, Jian C, Lin C, Li X. Panax notoginseng Saponins Attenuate Oxygen-Glucose Deprivation/Reoxygenation-Induced Injury in Human SH-SY5Y Cells by Regulating the Expression of Inflammatory Factors through miR-155. Biol Pharm Bull. 2019; 42(3): 462-467.

18. Zhang M, Guan Y, Xu J, Qin J, Li C, Ma X, Zhang Z, Zhang B, Tang J. Evaluating the protective mechanism of panax notoginseng saponins against oxidative stress damage by quantifying the biomechanical properties of single cell. Anal Chim Acta. 2019; 1048: 186-193.

19. Guo X, Sun W, Luo G, Wu L, Xu G, Hou D, Hou Y, Guo X, Mu X, Qin L, Liu T. Panax notoginseng saponins alleviate skeletal muscle insulin resistance by regulating the IRS1-PI3K-AKT signaling pathway and GLUT4 expression. FEBS Open Bio. 2019; 9(5): 1008-1019.

20. Chian S, Zhao Y, Xu M, Yu X, Ke X, Gao R, Yin L. Ginsenoside Rd reverses cisplatin resistance in nonsmall-cell lung cancer A549 cells by downregulating the nuclear factor erythroid 2-related factor 2 pathway. Anticancer Drugs. 2019; 30(8): 838-845.

21. Zhang $\mathrm{H}$, Gong $\mathrm{J}$, Zhang $\mathrm{H}$, Kong $\mathrm{D}$. Induction of apoptosis and reversal of permeability glycoproteinmediated multidrug resistance of MCF-7/ADM by ginsenoside Rh2. Int J Clin Exp Pathol. 2015; 8(5): 4444-56.

22. Li C, Sun BQ, Gai XD. Compounds from Chinese herbal medicines as reversal agents for Pglycoprotein-mediated multidrug resistance in tumours. Clin Transl Oncol. 2014; 16(7): 593-8.

23. Feng SL, Luo HB, Cai L, Zhang J, Wang D, Chen YJ, Zhan HX, Jiang ZH, Xie Y. Ginsenoside Rg5 overcomes chemotherapeutic multidrug resistance mediated by $A B C B 1$ transporter: in vitro and in vivo study. J Ginseng Res. 2020; 44(2): 247-257.

24. Lu Y, Yang R, Zhang H, Zhu X. The effects of Panax Notoginseng Saponins on P-glycoprotein and the function of glucocorticoid in lymphocytes of systemic lupus erythematosus patients. Chin $\mathrm{J}$ Of Rheumatology. 2011; 15: 38-41.

25. Xu Z, Ding WS, Lu Y. Effect of Panax notoginseng saponins on hormone resistance and lipid metabolism disorder in lupus nephritis mice. Zhejiang medicine. 2019; 41: 492-2496+2500. 
26. She Y, Wang N, Chen C, Zhu Y, Xia S, Hu C, Li Y. Effects of aluminum on immune functions of cultured splenic T and B lymphocytes in rats. Biol Trace Elem Res. 2012; 147(1-3): 246-50.

27. Akis N, Madaio MP. Isolation, culture, and characterization of endothelial cells from mouse glomeruli. Kidney Int. 2004; 65(6): 2223-7.

28. Lasser C, Eldh M, Lotvall J. Isolation and characterization of RNA-containing exosomes. J Vis Exp.2012; 9(59): e3037.

29. Zhu J, Liu B, Wang Z, Wang D, Ni H, Zhang L, Wang Y. Exosomes from nicotine-stimulated macrophages accelerate atherosclerosis through miR-21-3p/PTEN-mediated VSMC migration and proliferation. Theranostics. 2019; 9(23): 6901-6919.

30. Xiao J, Pan Y, Li XH, Yang XY, Feng YL, Tan HH, Jiang L, Feng J, Yu XY. Cardiac progenitor cellderived exosomes prevent cardiomyocytes apoptosis through exosomal miR-21 by targeting PDCD4. Cell Death Dis. 2016; 7(6): e2277.

31. Tsujimura S, Saito K, Nakayamada S, Nakano K, Tanaka Y. Clinical relevance of the expression of Pglycoprotein on peripheral blood lymphocytes to steroid resistance in patients with systemic lupus erythematosus. Arthritis Rheum. 2005; 52(6): 1676-83.

32. Xiao Y, Chen W, Chen R, Luo A, Chen D, Liang Q, Liu T, Chen X, Tan W. Exosomal MicroRNA Expression Profiling Analysis of the Effects of Lycium Barbarum Polysaccharide on Gestational Diabetes Mellitus Mice. Evid Based Complement Alternat Med. 2020; 2020: 2953502.

33. Watson ML, Rao JK, Gilkeson GS, Ruiz P, Eicher EM, Pisetsky DS, Matsuzawa A, Rochelle JM, Seldin MF. Genetic analysis of MRL-Ipr mice: relationship of the Fas apoptosis gene to disease manifestations and renal disease-modifying loci. J Exp Med. 1992; 176(6): 1645-56.

34. Ma X, Hu M, Wang H, Li J. Discovery of traditional Chinese medicine monomers and their synthetic intermediates, analogs or derivatives for battling P-gp-mediated multi-drug resistance. Eur J Med Chem. 2018; 159: 381-392.

35. Torreggiani E, Roncuzzi L, Perut F, Zini N, Baldini N. Multimodal transfer of MDR by exosomes in human osteosarcoma. Int J Oncol. 2016; 49(1): 189-96.

36. Bach DH, Hong JY, Park HJ, Lee SK. The role of exosomes and miRNAs in drug-resistance of cancer cells. Int J Cancer. 2017; 141(2): 220-230.

37. Lopes-Rodrigues V, Sousa E, Vasconcelos MH. Curcumin as a Modulator of P-Glycoprotein in Cancer: Challenges and Perspectives. Pharmaceuticals (Basel). 2016; 9(4): 71.

38. Liu C, Gong Q, Chen T, Lv J, Feng Z, Liu P, Deng Z. Treatment with 20(S)-ginsenoside Rg3 reverses multidrug resistance in A549/DDP xenograft tumors. Oncol Lett. 2018; 15(4): 4376-4382.

39. Zhou X, Wang A, Wang L, Yin J, Wang L, Di L, Hoi MP, Shan L, Wu X, Wang Y. A DanshensuTetramethylpyrazine Conjugate DT-010 Overcomes Multidrug Resistance in Human Breast Cancer. Front Pharmacol. 2019; 10: 722.

40. Wang H, Jia XH, Chen JR, Wang JY, Li YJ. Osthole shows the potential to overcome Pglycoprotein-mediated multidrug resistance in human myelogenous leukemia K562/ADM cells by inhibiting the PI3K/Akt signaling pathway. Oncol Rep. 2016; 35(6): 3659-68. 
Figures

A
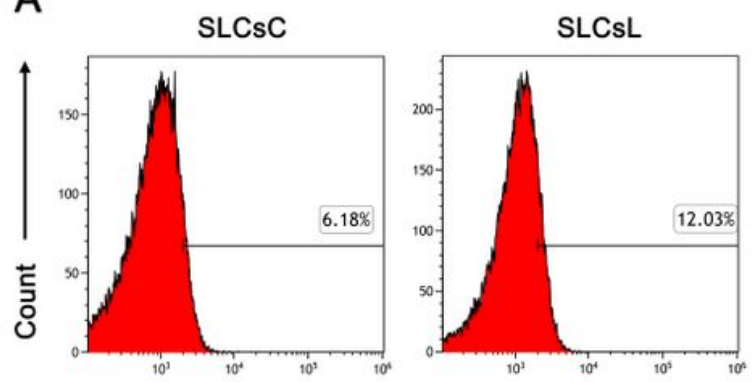

P-gp

C

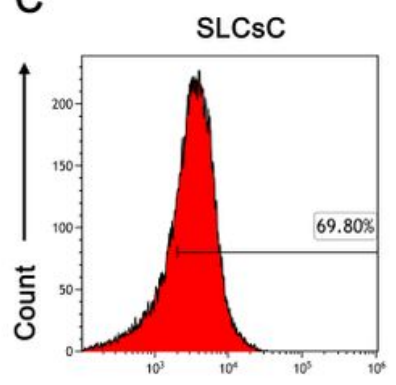

Rh-123
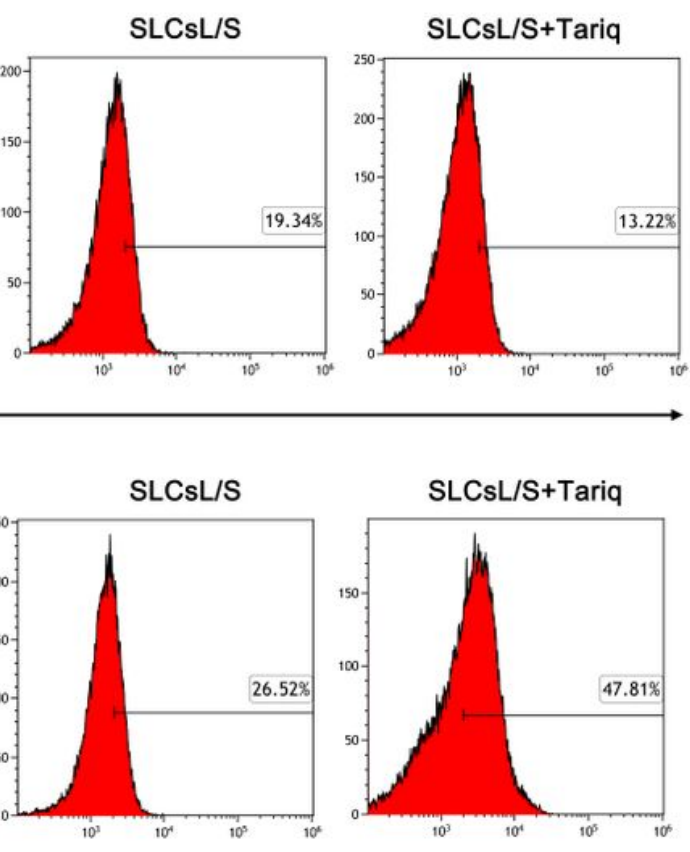

B

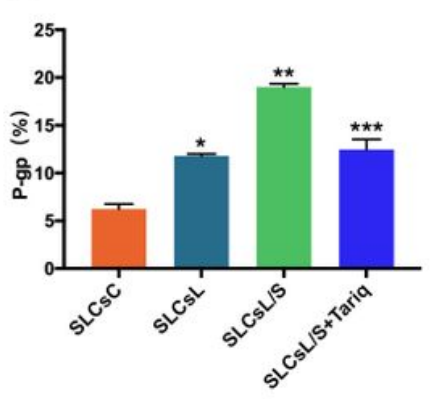

D

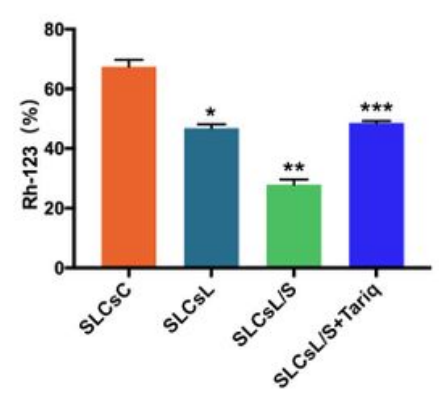

Figure 1

Effect of SLCsL/S on the SR of GECs. (A) P-gp expression in GECs was measured by flow cytometry. (B) Analysis of P-gp expression. (C) Rh-123 accumulation in GECs was measured by flow cytometry. (D) Analysis of the intracellular accumulation of Rh-123. Data were repeated three times and expressed as the mean \pm SD. ${ }^{*} P<0.05$ vs. SLCsC. ${ }^{*} P<0.05$ vs. SLCsL. ${ }^{* \star *} P<0.05$ vs. SLCsL/S. 
A

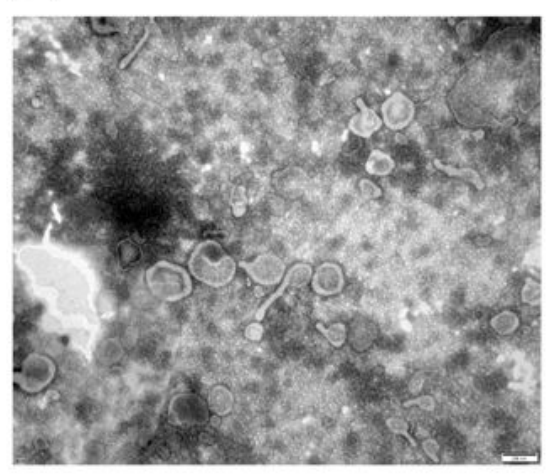

D
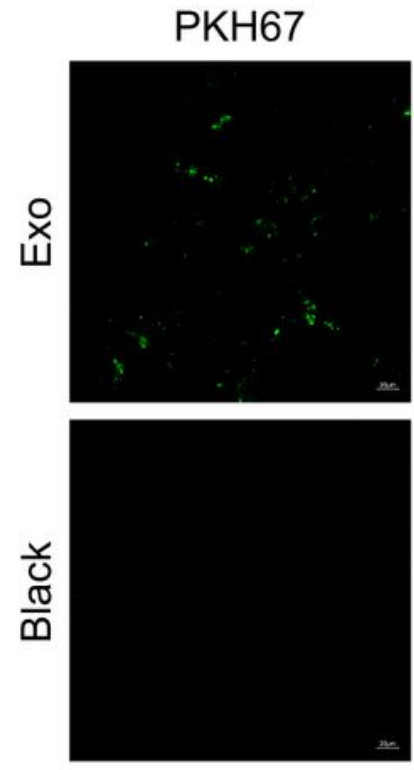

$E$
B

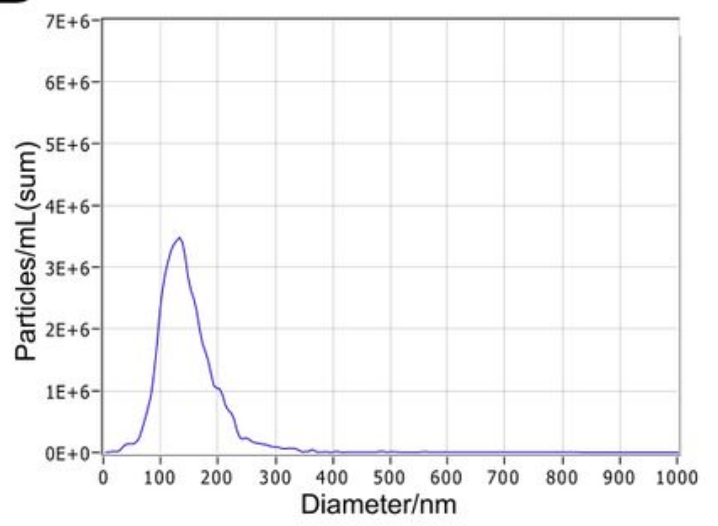

C

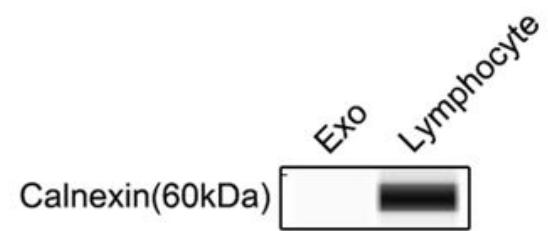

TSG101(54kDa) CD63(47kDa)
Phalloidin
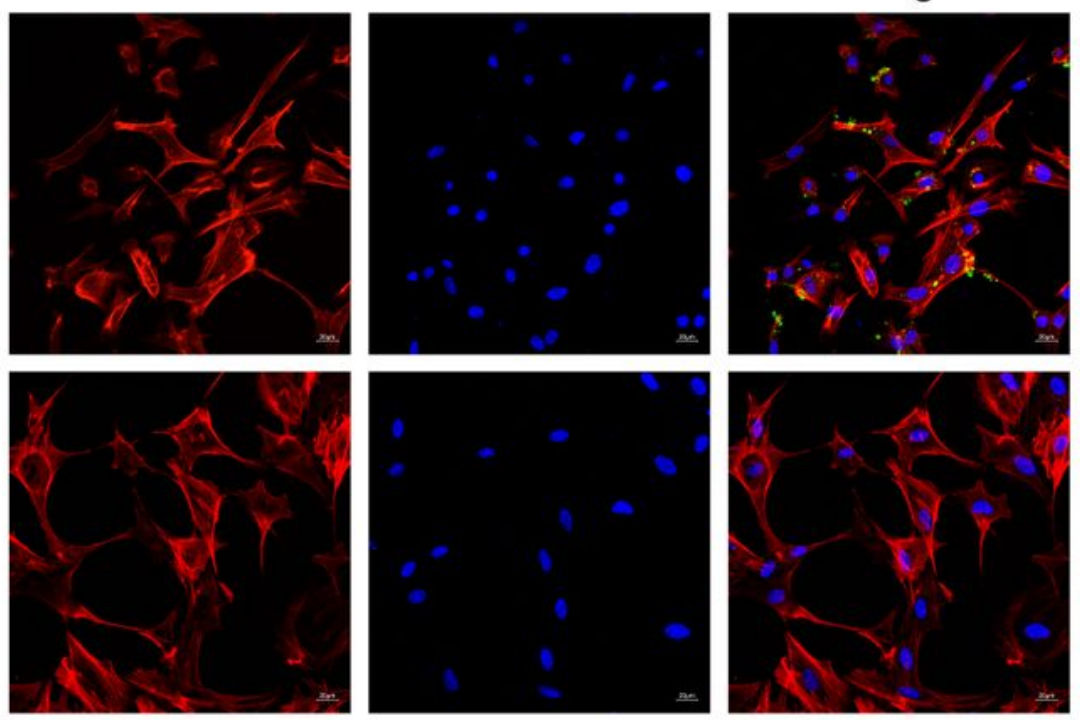

$\mathrm{F}$
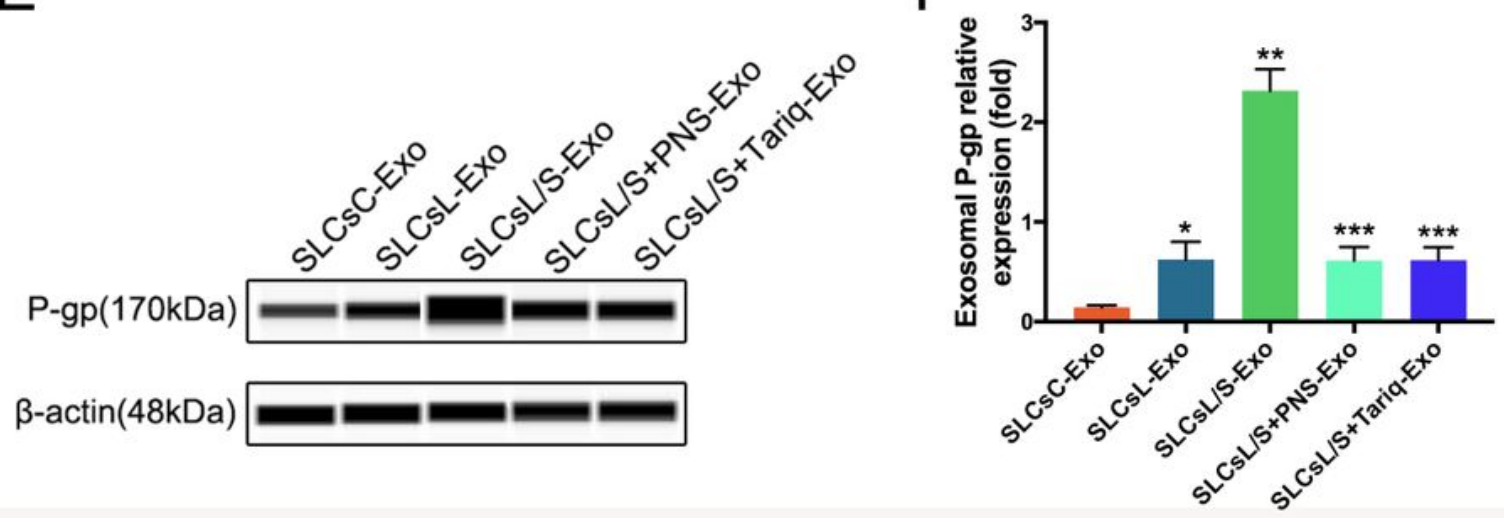

\section{Figure 2}

Internalization of lymphocyte-derived exosomes by GECs and the effect of PNS on exosomal P-gp expression. (A) Lymphocyte-derived exosomes were identified by TEM. Magnification: $\times 150,000$. Scale bar: $200 \mathrm{~nm}$. (B) The particle diameter (nm) of the exosomes. (C) Protein blot of exosomes extracted from lymphocytes using the calnexin antibody, CD63 antibody and TSG101 antibody. (D) The uptake of PKH67-labeled lymphocyte-exos was evident in GECs after $24 \mathrm{~h}$ of incubation. No stain was observed in 
the negative control condition. Scale bars: $20 \mu \mathrm{m}$. (E) P-gp expression in exosomes extracted from SLCsC, SLCsL, SLCsL/S, SLCsL/S+PNS and SLCsL/S+Tariq was measured by WB ( $\beta$-actin was used as an internal reference). (F) Analysis of P-gp expression. Data were repeated three times and expressed as the mean \pm SD. ${ }^{*} P<0.05$ vs. SLCsC-exo. ${ }^{*} P<0.05$ vs. SLCsL-exo. ${ }^{* *} P<0.05$ vs. SLCsL/S-exo.

A

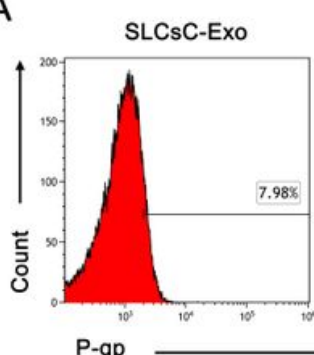

C

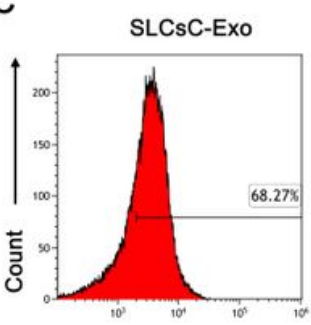

Rh-123
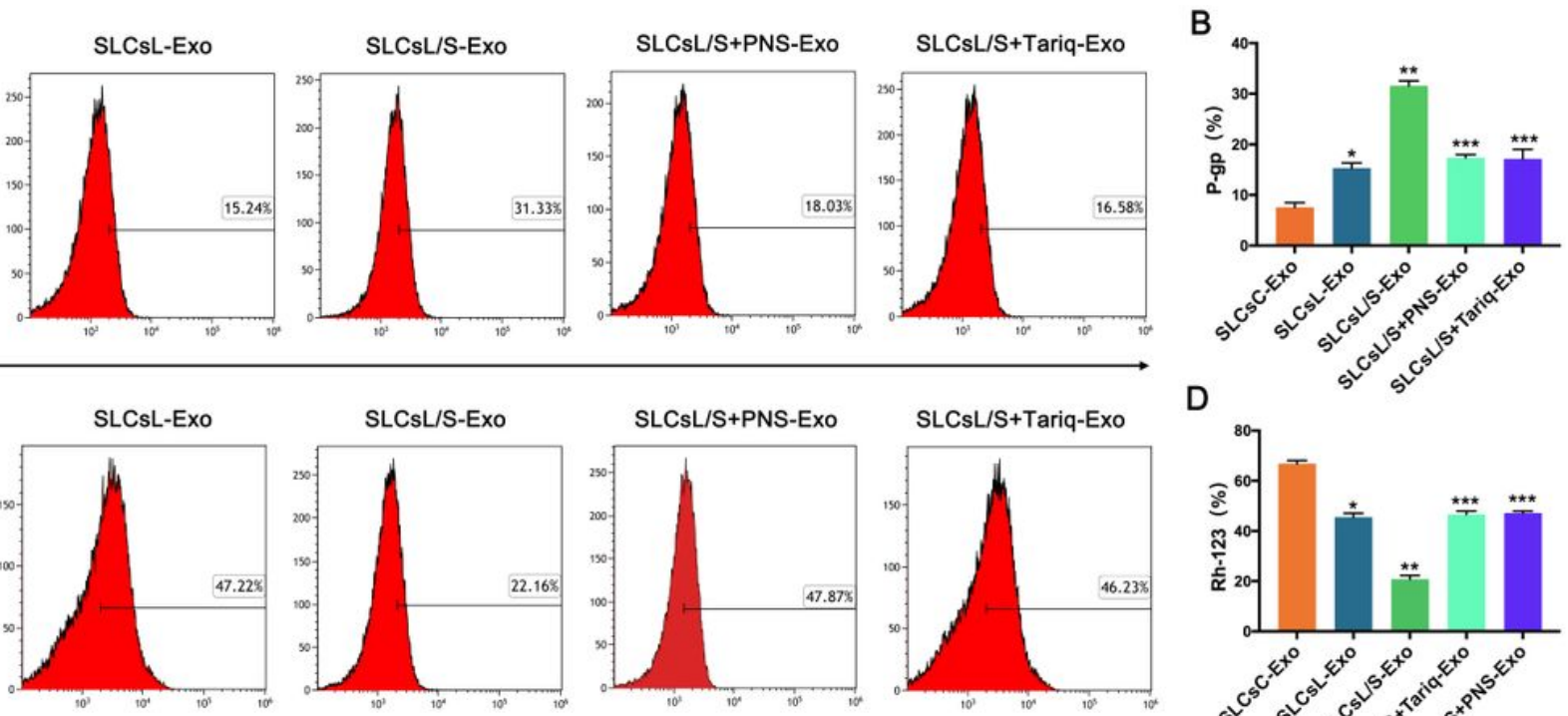

SLCsL/S+PNS-Exo
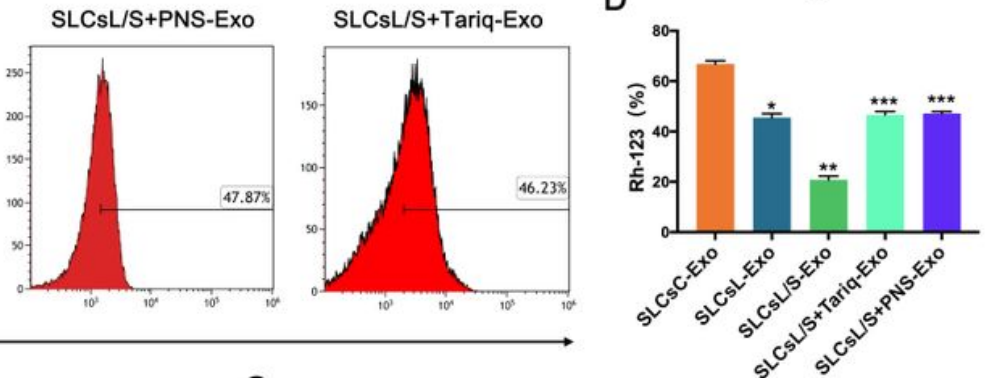

E

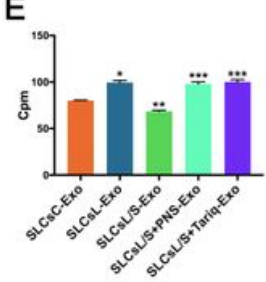

$\mathrm{F}$

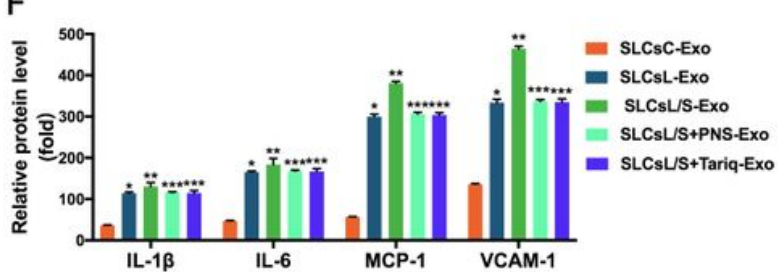

$\mathrm{H}$

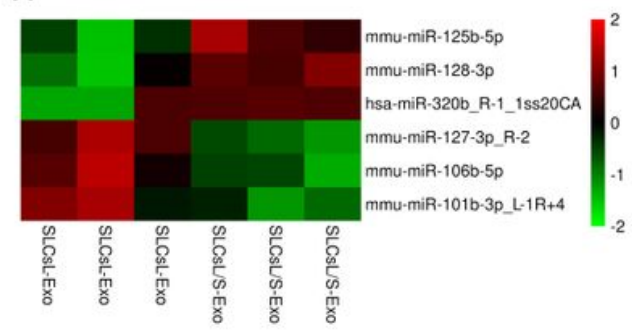

I

G

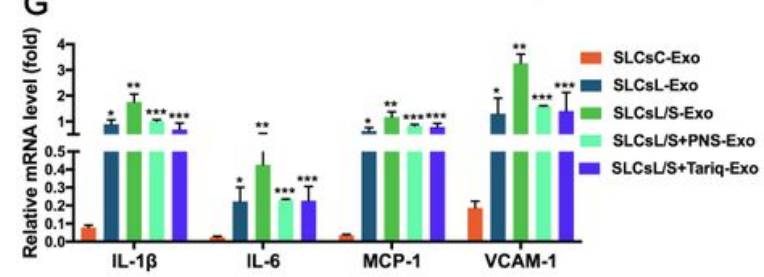

$J$
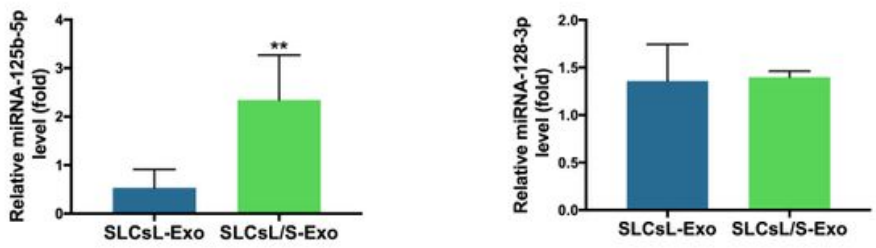

Figure 3

Effect of SLCsL/S-derived exosomes on SR and inflammatory injury in GECs and PNS intervention in vitro. (A) P-gp expression in GECs exposed to exosomes was measured by flow cytometry. (B) Analysis of P-gp expression. (C) Rh-123 accumulation in GECs exposed to exosomes was measured by flow cytometry. (D) Analysis of the intracellular accumulation of Rh-123. (E) Intracellular dexamethasone levels in GECs exposed to exosomes were evaluated by scintillation counter. $(F, G)$ Extracellular IL-1 $\beta$, IL-6, MCP-1 and VCAM-1 were determined after GECs were treated with exosomes for $48 \mathrm{~h}$ by ELISA and RTPCR. $(H)$ Heat map showing the significantly upregulated and downregulated miRNAs in the exosomes derived from GECs after treated with SLCsL-exo and SLCsL/S-exo. (I, J) RT-PCR validation of miRNAs in exosomes from GECs after treated with SLCsL-exo and SLCsL/S-exo. Data were repeated three times and expressed as the mean $\pm \mathrm{SD}$. ${ }^{*} \mathrm{P}<0.05$ vs. SLCsC-exo. ${ }^{*} \mathrm{P}<0.05$ vs. SLCsL-exo. ${ }^{*}{ }^{*} \mathrm{P}<0.05$ vs. $S L C s L / S-e x o$. 
A
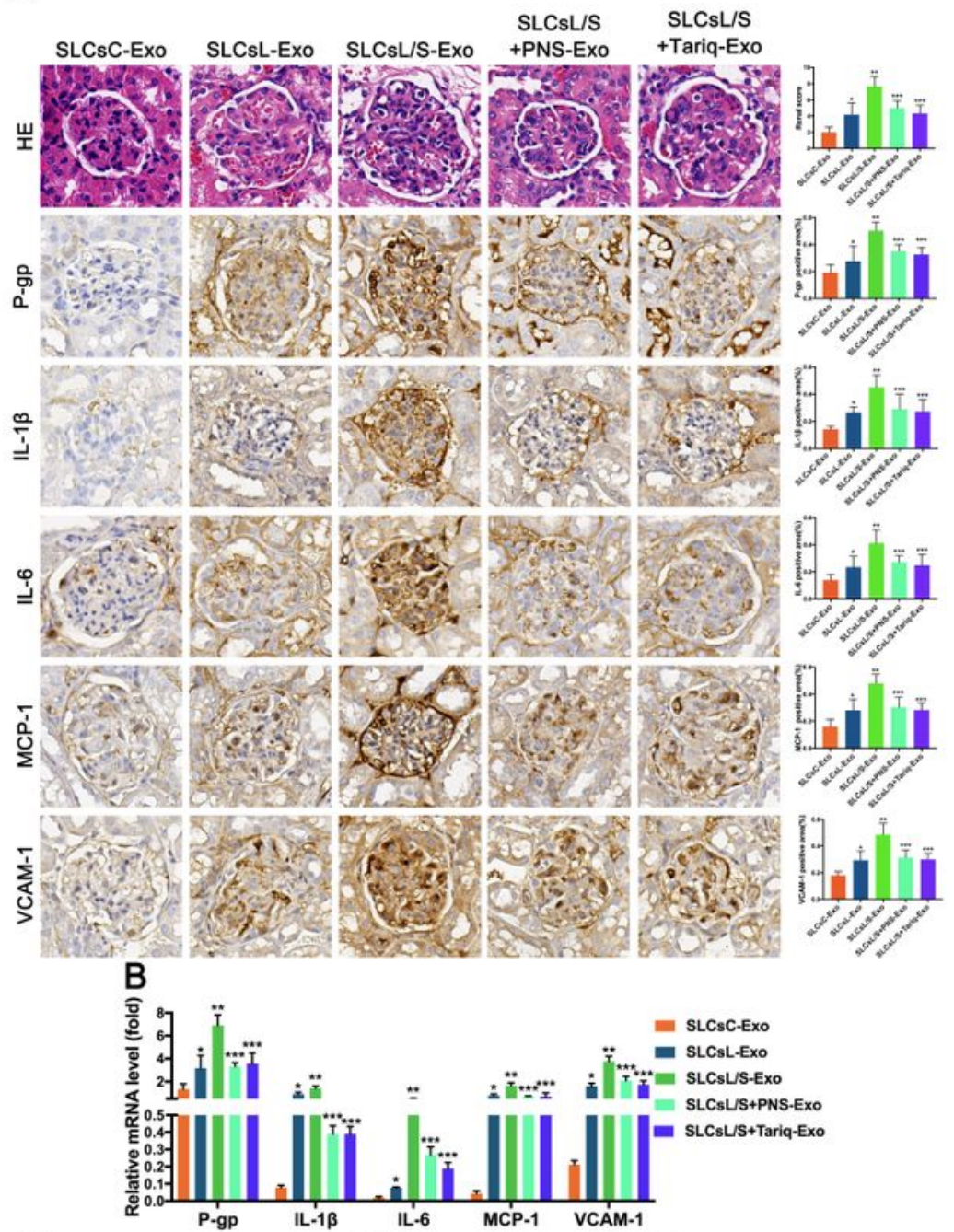

C
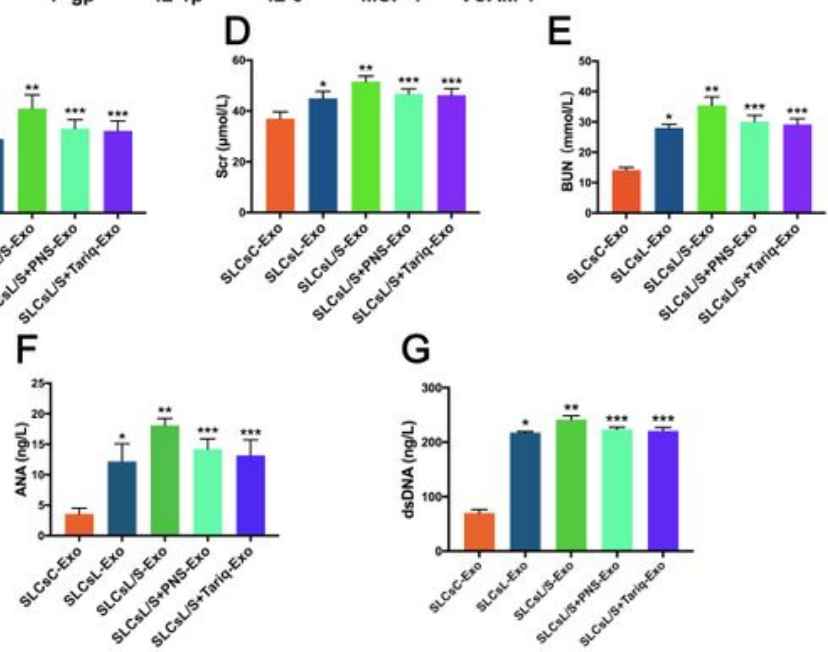

Figure 4

Effect of SLCsL/S-derived exosomes on SR and inflammatory injury in GECs and PNS intervention in

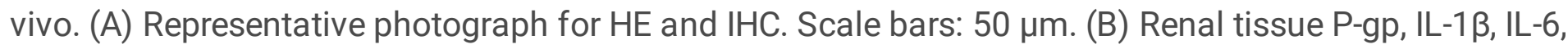
MCP-1 and VCAM-1 mRNA were determined by RT-PCR. (C, D, E, F, G) The levels of 24 h Upro, SCr, BUN, ANA and dsDNA. Data were repeated three times and expressed as the mean \pm SD. ${ }^{*} P<0.05$ vs. SLCsCexo. **P<0.05 vs. SLCsL-exo. ***P<0.05 vs. SLCsL/S-exo. 


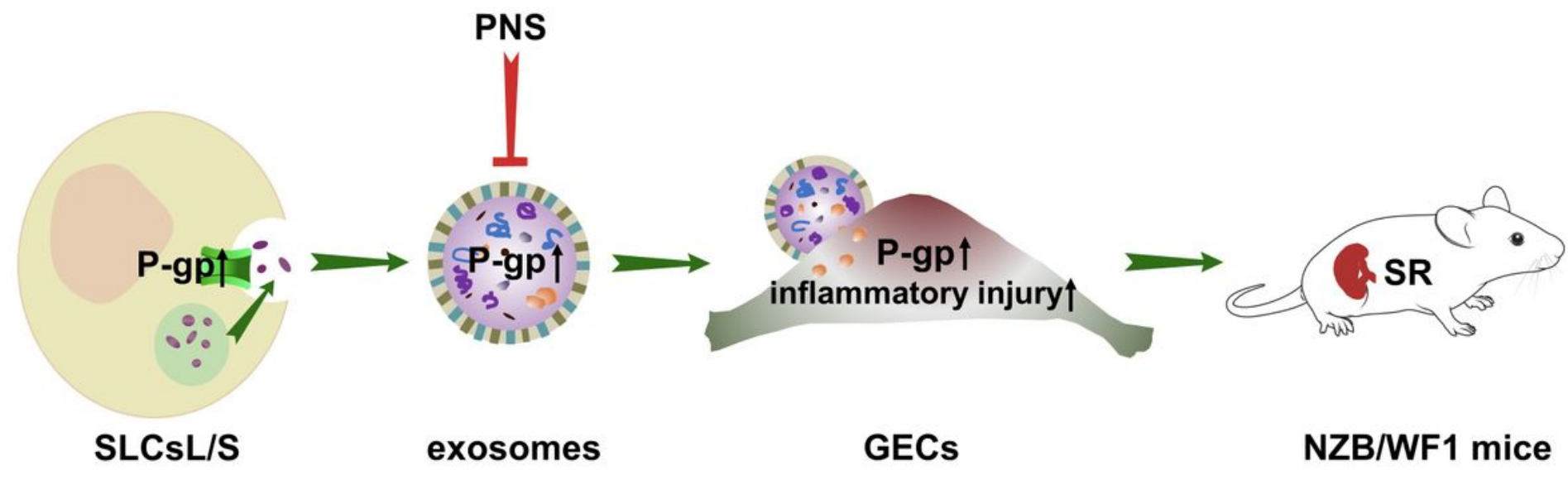

Figure 5

Schematic illustrations of putative signaling mechanisms of PNS in reversing the steroid resistance through reducing the content of P-gp in the exosomes transferred from SLCsL/S to GECs.

\section{Supplementary Files}

This is a list of supplementary files associated with this preprint. Click to download.

- Supplementaryfigures.doc

- SupportingDocumentsforWB.doc 